\begin{tabular}{|l|}
\hline Access this article online \\
\hline Quick Response Code: \\
\hline \\
Website: \\
www.Jlponline.org \\
\hline DOI: \\
10.4103/JLP.JLP_129_18 \\
\hline
\end{tabular}

Department of Microbiology, Government Medical College, Srinagar, Jammu and Kashmir, India

Address for correspondence:

Dr. Asifa Nazir, 370 BBC, Shivpora, Srinagar - 190004 , Jammu and Kashmir, India.

E-mail: asifanazir@gmail.

Submission: 03-10-2018 Accepted: 20-12-2018

\title{
Multidrug-resistant Acinetobacter septicemia in neonates: A study from a teaching hospital of Northern India
}

\author{
Asifa Nazir
}

\section{Abstract:}

BACKGROUND: Acinetobacter species are typical nosocomial pathogens causing infections and high mortality, almost exclusively in compromised hospitalized patients. Acinetobacter sp. are intrinsically less susceptible to antibiotics and have propensity to acquire resistance. Multidrug-resistant (MDR) Acinetobacter sp. blood infection in the neonatal intensive care unit patients create a great problem in hospital settings.

AIMS: A prospective data analysis was performed over a one year period of all neonates admitted with sepsis who developed Acinetobacter infection and their antibiotic susceptibility pattern was carried out.

MATERIALS AND METHODS: Blood samples of infected neonates were collected aseptically and cases of Acinetobacter septicemia were identified. Speciation of Acinetobacter species was done. Various risk factors were identified and their drug-sensitivity test was performed.

RESULTS: The incidence of neonatal septicemia due to Acinetobacter species was $13.7 \%$ (49/357). Predominant species isolated was Acinetobacter baumannii (98\%). The major symptoms were lethargy and poor feeding. The major signs were tachypnea, intercostal retraction, and respiratory distress. The major fetal risk factors were low birth weight and prematurity. High degree of resistance was observed to the various antibiotics used. Majority of the isolates (95.9\%) were MDR while $93.68 \%$ were resistant to carbapenems as well as extensively drug resistant. However, all the strains were sensitive to colistin.

CONCLUSION: MDR Acinetobacter septicemia in neonatal patients is becoming alarmingly frequent and is associated with significant mortality and morbidity. Therefore, rational antibiotic use is mandatory along with an effective infection control policy in neonatal intensive care areas of each hospital to control Acinetobacter infection and improve outcome.

Key words:

Acinetobacter septicemia, blood culture, multidrug resistance, neonatal sepsis

\section{Introduction}

A cinetobacter species are aerobic Gram-negative, catalase-positive, oxidase-negative coccobacilli which were first described in 1911.[1] Members of the genus Acinetobacter are ubiquitous, free-living organisms that prefer moist environment and can be easily obtained from soil, water, food, and sewage. ${ }^{[2]}$ They are nonfermentative organisms that have

This is an open access journal, and articles are distributed under the terms of the Creative Commons Attribution-NonCommercial-ShareAlike 4.0 License, which allows others to remix, tweak, and build upon the work non-commercially, as long as appropriate credit is given and the new creations are licensed under the identical terms.

For reprints contact: reprints@medknow.com emerged as significant nosocomial pathogens in the hospital setting and are responsible for intermittent outbreaks. ${ }^{[3]}$ According to the National Neonatal Perinatal Database 2000, the incidence of neonatal septicemia in India has been reported to be $24 / 1000$ per live births. Acinetobacter sp. are gaining importance as a potential pathogen in neonatal septicemia because of its frequent isolation and multidrug resistance (MDR). ${ }^{[4]}$ Acinetobacter species are the second most commonly isolated nonfermenter organisms in human specimens (Pseudomonas aeruginosa

How to cite this article: Nazir A. Multidrug-resistant Acinetobacter septicemia in neonates: A study from a teaching hospital of Northern India. J Lab Physicians 2019;11:23-8. 
is the most common). ${ }^{[5]}$ They rank fourth (after P. aeruginosa, Staphylococcus aureus, and Klebsiella pneumoniae) among the most frequent hospital-acquired infectious agents. ${ }^{[6]}$

According to the Centers for Disease Control and Prevention (CDC), the species Acinetobacter baumannii accounts for nearly $80 \%$ of reported Acinetobacter infections. ${ }^{[7]} \mathrm{A}$. baumannii has become an increasingly frequent cause of healthcare-associated infections (HAIs), particularly in intensive care units (ICUs). ${ }^{[8,9]}$

Prior antibiotic use, prolonged hospitalization, colonization pressure, and enteral feeding have all been implicated in risk of Acinetobacter infection. ${ }^{[10,11]}$ In recent years, Acinetobacter has been increasingly recognized as a significant healthcare-associated, opportunistic, MDR pathogen, and the rate of isolation has increased since the last two decades worldwide with a high morbidity and a high mortality rate, especially in immunocompromised patients ranging from $26.5 \%$ to $91 \% .^{[3,12]}$

Prolonged survival in the healthcare environment-along with MDR - colonization potential, and contact transmission (hands, instruments, and equipment) - is some of the challenging factors in Acinetobacter prevention and control. ${ }^{[13]}$

Antimicrobial resistance among Acinetobacter species has increased substantially in the past decade. ${ }^{[14]}$ The capacity of Acinetobacter species for extensive antimicrobial resistance may be due in part to the organism's relatively impermeable outer membrane and its environmental exposure to a large reservoir of resistance genes. ${ }^{[15]}$ Further, carbapenem resistance is increasingly reported and has become a significant public health concern. ${ }^{[16,17]}$

Different terms such as "MDR," "extensive drug resistant (XDR)," and "pandrug resistant (PDR)" have been used with varied definitions to describe the extent of antimicrobial resistance among Acinetobacter sp., but there is no accepted definition for the extent of resistance in the bacteria. ${ }^{[18,19]}$

A group of international experts came together through a joint initiative by the European Centre for Disease Prevention and Control ${ }^{[20]}$ and the $\mathrm{CDC}$, to create a standardized international terminology with which to describe acquired resistance profiles in $S$. aureus, Enterococcus spp., Enterobacteriaceae (other than Salmonella and Shigella), P. aeruginosa, and Acinetobacter spp., all bacteria often responsible for HAIs and prone to MDR. MDR was defined as acquired nonsusceptibility to at least one agent in three or more antimicrobial categories, XDR was defined as nonsusceptibility to at least one agent in all but two or fewer antimicrobial categories (i.e., bacterial isolates remain susceptible to only one or two categories), and PDR was defined as nonsusceptibility to all agents in all antimicrobial categories. A review was recently published ${ }^{[21]}$ in which the definitions were proposed for grading various antimicrobial resistance profiles.

In the present study "MDR Acinetobacter sp." would be defined as the isolate resistant to at least three classes of antimicrobial agents such as all penicillins and cephalosporins (including inhibitor combinations), fluroquinolones, and aminoglycosides. "XDR Acinetobacter spp." would be the Acinetobacter sp. isolate that is resistant to the three classes of antimicrobials described above (MDR) and shall also be resistant to carbapenems. Finally, "PDR Acinetobacter spp." would be the XDR Acinetobacter spp. that is resistant to polymyxins and tigecycline. ${ }^{[22]}$

Since very few studies have been published on bacteremia caused by Acinetobacter species in our region, this study was undertaken to determine the incidence and antibiotic resistance pattern of Acinetobacter species isolated from blood samples of neonatal septicemia patients.

\section{Materials and Methods}

This prospective study was conducted in the Department of Microbiology of a teaching hospital of Northern India over a period of 1 year. The study was approved by the ethical committee of our institute. All blood culture positive for Acinetobacter sp. and their in vitro sensitivity to antibiotics were included from March 2017 to February 2018. Bacteremia was defined as the presence of Acinetobacter sp. in one or more blood cultures from a patient during hospitalization.

Blood was collected from suspected cases of neonatal sepsis by maintaining strict aseptic precaution in blood culture bottles and sent immediately to Microbiology Laboratory. Blood specimens were cultured using BacTAlert3D (BioMerieux, India ${ }^{\circledR}$ ) automated blood culture system. $1 \mathrm{ml}$ of blood was inoculated into ready to use BacT/ALERT PF Plus culture bottles (yellow coded) for pediatric use with all due precautions and shaken well. Positive or negative culture bottles were determined by BacT / ALERT microbial detection system. Blood cultures were considered negative only after 7 days of incubation.

Gram stain was carried out on positive bottles, followed by inoculation onto blood agar and MacConkey agar plates, and incubated aerobically for $24 \mathrm{~h}$ at $37^{\circ} \mathrm{C}$. Identification was carried up to species level, and antimicrobial susceptibility testing was done with an automated microbiology system (Vitek 2 Compact 
60 System, BioMerieux India ${ }^{\circledR}$ ) and interpreted according to the Clinical and Laboratory Standards Institute criteria. ${ }^{[23]}$ The patient's data that were collected included age, sex, underlying diseases, and risk factors. Quality control was performed by testing these same antimicrobials against reference strains of bacteria.

Statistical analysis was done to see the association between various risk factors and Acinetobacter septicemia.

\section{Results}

A total of 357 blood culture samples were consecutively included in the study. Acinetobacter sp. was isolated from $49(13.7 \%)$ samples out of which 48 (98\%) were A. baumannii, 1 (0.02\%) was Acinetobacter lwoffii.

Among 357 cases included in the study, the male-to-female ratio was 2:1. The gestational age was $<36$ weeks in $38(77.5 \%)$ cases and low birth weight babies were $40(81.6 \%)$.

The main risk factors associated with Acinetobacter septicemia were low birth weight $(81.6 \%)$, prematurity $(77.5 \%)$, prolonged intravenous antibiotic use $(75.5 \%)$, mechanical ventilation $(46.9 \%)$, and prolonged hospital stay (44.8\%) [Table 1].

The major signs and symptoms included poor feeding/activity $(81.6 \%)$ followed by respiratory distress (57.1\%), convulsions (12.2\%), and fever (12.2\%). Jaundice was seen in $6.12 \%$ and abdominal distension in $4.08 \%$ cases [Table 2].

Antibiotic-susceptibility pattern was studied among the Acinetobacter isolates [Table 3]. High degree of resistance was observed to the various antibiotics used.

Majority of the isolates (95.9\%) were MDR, i.e., resistant to penicillins and cephalosporins (including inhibitor combinations), fluoroquinolones, and aminoglycosides while $93.68 \%$ were resistant to carbapenems as well (XDR). Minocycline resistance was seen in 69.3\% cases while tigecycline resistance was observed in $6 \%$ cases. However, all the strains were sensitive to colistin [Table 4].

\section{Discussion}

Over the last three decades, Acinetobacter species has emerged as an important pathogen in the healthcare setting, both globally and locally. It has remarkable ability to develop or acquire multiple antibiotic resistance and propensity to survive for prolonged periods under a wide range of environmental conditions, making it a
Table 1: Potential risk factors identified in cases of Acinetobacter septicemia neonates $(n=49)$

\begin{tabular}{lc}
\hline Risk factors & Number of cases $(\%)$ \\
\hline Low birth weight & $40(81.6)$ \\
Prematurity & $38(77.5)$ \\
Prolonged IV antibiotics & $37(75.5)$ \\
Ventilator support & $23(46.9)$ \\
Prolonged NICU stay (>7 days) & $22(44.8)$ \\
\hline
\end{tabular}

$\mathrm{IV}=$ Intravenous, $\mathrm{NICU}=$ Neonatal intensive care unit

Table 2: Clinical presentations observed in cases of Acinetobacter septicemia neonates $(n=49)$

\begin{tabular}{lc}
\hline Clinical signs and symptoms & Number of cases (\%) \\
\hline Poor activity/poor feeding & $40(81.6)$ \\
Respiratory distress & $28(57.1)$ \\
Convulsions & $6(12.2)$ \\
Fever & $6(12.2)$ \\
Jaundice & $3(6.12)$ \\
Abdominal distension & $2(4.08)$ \\
\hline
\end{tabular}

Table 3: Susceptibility pattern of Acinetobacter spp. to different antimicrobial agents $(n=49)$

\begin{tabular}{lcc}
\hline Antibiotic & Sensitive (\%) & Resistant (\%) \\
\hline Ceftriaxone & $0(0)$ & $49(100)$ \\
Cefepime & $0(0)$ & $49(100)$ \\
Gentamicin & $2(4.08)$ & $47(95.9)$ \\
Ciprofloxacin & $2(4.08)$ & $47(95.9)$ \\
Imipenem & $3(4.08)$ & $46(93.8)$ \\
Meropenem & $2(4.08)$ & $47(95.9)$ \\
Piperacillin + tazobactam & $1(2.04)$ & $48(97.9)$ \\
Ceftriaxone + sulbactam & $2(4.08)$ & $47(95.9)$ \\
Ampicillin & $0(0)$ & $49(100)$ \\
Amoxicillin clavulanic acid & $0(0)$ & $49(100)$ \\
Minocycline & $15(30.6)$ & $34(69.3)$ \\
Tigecycline & $43(87)$ & $6(12.2)$ \\
Colistin & $49(100)$ & $0(0)$ \\
\hline
\end{tabular}

Table 4: Rate of multidrug-resistant, extensive drug-resistant and pan drug-resistant Acinetobacter spp. $(n=49)$

\begin{tabular}{llc}
\hline $\begin{array}{l}\text { Categories of } \\
\text { Acinetobacter }\end{array}$ & Definition & $n(\%)$ \\
\hline MDR & $\begin{array}{l}\text { Resistant to three groups } \\
\text { Penicillins and cephalosporins } \\
\text { (including inhibitor combinations) } \\
\text { Fluoroquinolones } \\
\end{array}$ & $47(95.9)$ \\
& Aminoglycosides & \\
XDR & MDR + resistant to carbapenems & $46(93.8)$ \\
PDR & XDR + resistant to polymyxin E (colistin) & 0 \\
\hline $\begin{array}{l}\text { MDR = Multidrug resistant, XDR = Extensive drug resistant, PDR = Pan drug } \\
\text { resistant }\end{array}$
\end{tabular}

frequent cause of hospital outbreaks and an endemic healthcare-associated pathogen. It commonly targets the most vulnerable hospitalized and critically ill patients with breaches in skin integrity who require airway protection, causing pneumonia, urinary tract infection, wound infection, and bacteremia. ${ }^{[9-11]}$ 
MDR nosocomial Acinetobacter sp. septicemia may cause severe clinical diseases in neonate that is associated with a high mortality. ${ }^{[22]}$

The present study was undertaken to find the incidence of Acinetobacter septicemia in neonates and its antibiotic resistance pattern.

In our study, the Acinetobacter sepsis incidence was $13.7 \%$. This percentage was similar to the studies which were conducted by Vinodkumar and Neelagund $^{[4]}(8.3 \%)$, Arora and Jaitwani ${ }^{[24]}(12.3 \%)$, and Mondal et al. (15.2\%). ${ }^{[25]}$ A. baumannii was the predominant species in our study $(98 \%)$. In other studies, also, the main species responsible for neonatal sepsis was A. baumannii. ${ }^{[25]}$ This percentage was however higher than the percentage in the study which was conducted by Arora and Jaitwani, (56.52\%). ${ }^{[24]}$ Female sex was affected more commonly with ratio of 1.6:1 in our study which is similar to the finding by Christo et al. ${ }^{[26]}$

In our study, Acinetobacter sepsis was found more in premature $(77.5 \%)$ and very low birth weight babies $(81.6 \%)$ which is similar to findings from other studies. ${ }^{[4,27,28]}$ Preterm infants have a 3-10-fold higher incidence of infection than full-term infants as they often require prolonged intravenous access, endotracheal intubation, or other invasive procedures that provide a portal of entry for infection. ${ }^{[29-32]}$ Outbreaks of Acinetobacter infection have been traced to medical equipment, emphasizing the need for special attention to disinfection of shared items and extra caution with respiratory care and wound care procedures. ${ }^{[33]}$ In our study, a significant association between Acinetobacter bloodstream infection and prolonged ICU stay was noted. Acinetobacter spp. has the reputation of causing outbreaks in ICUs. ${ }^{[34]}$ It indirectly predicts that the isolate is of hospital origin and poses a great threat for the neonate and rises a concern in the management. The organism can survive on environmental surfaces for months, making nosocomial transmission extremely difficult to prevent and control. ${ }^{[35]}$

MDR Acinetobacter has been reported worldwide and is now recognized as one of the most difficult HAIs. Mechanisms of acquiring resistance to cephalosporins and carbapenems described for $A$. baumannii are altered penicillin-binding proteins, presence of metallo-beta lactamases, and loss of porins. ${ }^{[15,16]}$

Acinetobacter strains isolated during our study exhibited MDR pattern. 95.9\% strains were MDR, i.e., resistant to penicillins and cephalosporins (including inhibitor combinations), fluoroquinolones, and aminoglycosides, while $93.68 \%$ were resistant to carbapenems as well (XDR).
A study conducted in New Delhi, India (2006), revealed that the prevalence of carbapenem resistance in Acinetobacter spp. isolated from different clinical samples was found to be almost 35\%. ${ }^{[36]}$ Further, Gladstone et al. from Vellore, India (2005), reported a prevalence of $14 \%$ carbapenem-resistant Acinetobacter spp., isolated from tracheal aspirates $(n=56) \cdot{ }^{[37]}$ In a surveillance study of the antibiotic susceptibility patterns of the isolates from the ICUs of five European countries (1999), the prevalence of resistance in Acinetobacter spp. to gentamicin was $0 \%-81 \%$, amikacin $10 \%-51 \%$, ciprofloxacin $19 \%-81 \%$, ceftazidime $0 \%-81 \%$, piperacillin-tazobactam $36 \%-75 \%$, and imipenem $5 \%-19 \%$. ${ }^{[38]}$

The Meropenem Yearly Susceptibility Test Information Collection (MYSTIC) program reported the antimicrobial susceptibility of 490 A. baumannii strains collected in 37 centers in 11 European countries from 1997 to 2000. ${ }^{[39]}$

Imipenem and meropenem were found as the most active agents against $A$. baumannii, with resistance rates of 16 and $18 \%$, respectively.

Subsequent data from 40 centers in 12 countries participating in the MYSTIC program (2006) revealed a substantial increase in resistance rates for meropenem (43.4\%) and imipenem (42.5\%). ${ }^{[40]}$

Data of the antibiotic susceptibilities of Acinetobacter from different geographical regions revealed that the resistance of Acinetobacter spp. to imipenem rose from no resistance to $40 \%(2000-2004) \cdot{ }^{[41]}$ The prevalence of imipenem resistance in A. baumannii isolated from a burns unit of the USA was found to be as high as $8 \%$ (2007). ${ }^{[42]}$ Resistances to major antimicrobial drugs as well as disinfectants are the major factors that make it a successful and persistent hospital pathogen. ${ }^{[30]}$

Many neonates in hospitals in India are now treated with carbapenems as a first-line therapy for sepsis or presumed sepsis. Against this backdrop, the widespread availability and antimicrobial use in community settings and the contribution of antimicrobial resistance as a complicating factor in neonate sepsis become extremely important.

In the present study, minocycline resistance was seen in $69.3 \%$ cases while tigecycline resistance was observed in $6 \%$ cases. Tigecycline is a minocycline derivative with enhanced in vitro activity against both Gram-positive and Gram-negative bacteria, including A. baumannii. However, clinical data in treating $A$. baumannii infections remain limited. Breakthrough bacteremia by $A$. baumannii in patients receiving tigecycline has also been reported. ${ }^{[43]}$ It is important to note that the emergence of tigecycline resistance may occur while the patient is on treatment. ${ }^{[44]}$ 
All the strains that we tested were sensitive to colistin. This is likely due to the fact that colistin has been limited from being used during the last several decades due to nephrotoxicity. At present, the therapeutic options for infections caused by antibiotic-resistant strains of $A$. baumannii are limited. This indicates that we should be judicious in use of antibiotics while treating Acinetobacter infections.

Acinetobacter sp. are rapidly spreading with emergence of extended resistance to even newer antimicrobials. They have the ability to acquire resistance at a much faster than another Gram-negative organism. ${ }^{[22]}$ Due to their ease of survival in the hospital environment, they have immense potential to cause nosocomial outbreaks. The global emergence of MDR A. baumannii has reduced the number of clinically available antibiotics that retain activity against this pathogen. For this reason, the development of novel treatment strategies for infections caused by $A$. baumannii is necessary.

\section{Conclusion}

Because of the tremendous challenge posed by MDR A. baumannii and the emergence and dissemination of mechanisms of resistance to any existing agent, solutions beyond the paradigm of antibiotics should be explored aggressively. ${ }^{[45]}$ Infection control is extremely important especially given the ability of $A$. baumannii to cause outbreaks. Contact precautions, hand-washing, and alcohol-hand decontamination, although universally encouraged, are seldom applied rigorously. Their importance, however, cannot be overstressed. ${ }^{[46]}$

There is an urgent need to enforce infection control measures and antimicrobial stewardship programs to prevent the further spread of these resistant Acinetobacter species and to delay the emergence of increased resistance in the bacteria.

\section{Financial support and sponsorship \\ Nil.}

\section{Conflicts of interest}

There are no conflicts of interest.

\section{References}

1. Towner KJ. Acinetobacter: An old friend, but a new enemy. J Hosp Infect 2009;73:355-63.

2. Gerner-Smidt P. Taxonomy and epidemiology of Acinetobacter infections. Rev Med Microbiol 1995;6:186-97.

3. Peleg AY, Seifert H, Paterson DL. Acinetobacter baumannii: Emergence of a successful pathogen. Clin Microbiol Rev 2008;21:538-82.

4. Vinodkumar CS, Neelagund YF. Acinetobacter septicaemia in neonates. Indian J Med Microbiol 2004;22:71.
5. Koneman EW, Allen SD, Janda WM, Schreckenberger PC, Winn WC Jr. Color Atlas and Textbook of Diagnostic Microbiology. $5^{\text {th }}$ ed. Philadelphia, USA: Lippincott Williams and Wilkins; 1997. p. 253-320.

6. Aktas O, Ozbek A. Prevalence and in vitro antimicrobial susceptibility patterns of Acinetobacter strains isolated from patients in intensive care units. J Int Med Res 2003;31:272-80.

7. Centers for Disease Control and Prevention. Overview of drug-resistant Acinetobacter Infections in Healthcare Settings. Available from: http://www.cdc.gov/ncidod/dhqp/ar acinetobacter.html. [Last accessed on 2009 Oct 26].

8. Sinha N, Agarwal J, Srivastava S, Singh M. Analysis of carbapenem-resistant Acinetobacter from a tertiary care setting in North India. Indian J Med Microbiol 2013;31:60-3.

9. Bergogne-Berezin E, Tower KJ. Acinetobacter sp. as nosocomial pathogens: Microbiological, clinical, and epidemiological features. Clin Microbial Rev 1996;9:148-65.

10. Fournier PE, Richet H. The epidemiology and control of Acinetobacter baumannii in health care facilities. Clin Infect Dis 2006;42:692-9.

11. Joly-Guillou ML. Clinical impact and pathogenicity of Acinetobacter. Clin Microbiol Infect 2005;11:868-73.

12. Punpanich W, Nithitamsakun N, Treeratweeraphong V, Suntarattiwong P. Risk factors for carbapenem non-susceptibility and mortality in Acinetobacter baumannii bacteremia in children. Int J Infect Dis 2012;16:e811-5.

13. Turkoglu M, Mirza E, Tunçcan ÖG, Erdem GU, Dizbay M, Yağc1 M, et al. Acinetobacter baumannii infection in patients with hematologic malignancies in intensive care unit: Risk factors and impact on mortality. J Crit Care 2011;26:460-7.

14. Lockhart SR, Abramson MA, Beekmann SE, Gallagher G, Riedel S, Diekema DJ, et al. Antimicrobial resistance among gram-negative bacilli causing infections in intensive care unit patients in the United States between 1993 and 2004. J Clin Microbiol 2007;45:3352-9.

15. Bonomo RA, Szabo D. Mechanisms of multidrug resistance in Acinetobacter species and Pseudomonas aeruginosa. Clin Infect Dis 2006;43 Suppl 2:S49-56.

16. Gordon NC, Wareham DW. Multidrug-resistant Acinetobacter baumannii: Mechanisms of virulence and resistance. Int J Antimicrob Agents 2010;35:219-26.

17. Poirel L, Nordmann P. Carbapenem resistance in Acinetobacter baumannii: Mechanisms and epidemiology. Clin Microbiol Infect 2006;12:826-36.

18. Falagas ME, Koletsi PK, Bliziotis IA. The diversity of definitions of multidrug-resistant (MDR) and pandrug-resistant (PDR) Acinetobacter baumannii and Pseudomonas aeruginosa. J Med Microbiol 2006;55:1619-29.

19. Falagas ME, Karageorgopoulos DE. Pandrug resistance (PDR), extensive drug resistance (XDR), and multidrug resistance (MDR) among gram-negative bacilli: Need for international harmonization in terminology. Clin Infect Dis 2008;46:1121-2.

20. European Centre for Disease Prevention and Control/European Medicines Agency. ECDC/EMEA Joint Technical Report. The Bacterial Challenge: Time to React. Stockholm, Sweden \& London, United Kingdom: European Centre for Disease Prevention \& Control and European Medicines Agency; 2009.

21. Magiorakos AP, Srinivasan A, Carey RB, Carmeli Y, Falagas ME, Giske CG, et al. Multidrug-resistant, extensively drug-resistant and pandrug-resistant bacteria: An international expert proposal for interim standard definitions for acquired resistance. Clin Microbiol Infect 2012;18:268-81.

22. Manchanda V, Sanchaita S, Singh N. Multidrug resistant Acinetobacter. J Glob Infect Dis 2010;2:291-304.

23. Clinical and Laboratory Standards Institute. Performance Standards for Antimicrobial Susceptibility Testing; Twenty-Third Informational Supplement. Clinical and Laboratory Standards 
Institute; M100-S23. January, 2013.

24. Arora U, Jaitwani J. Acinetobacter spp. - An emerging pathogen in neonatal septicemia in Amritsar. Indian J Med Microbiol 2006;24:81.

25. Mondal GP, Raghavan M, Bhat BV, Srinivasan S. Neonatal septicaemia among inborn and outborn babies in a referral hospital. Indian J Pediatr 1991;58:529-33.

26. Christo GG, Shenoy V, Matthai J, Shivananda PG, Venkatesh A. Acinetobacter sepsis in neonates. Indian Pediatr 1993;30:1413-6.

27. Mishra A, Mishra S, Jaganath G, Mittal RK, Gupta PK, Patra DP, et al. Acinetobacter sepsis in newborns. Indian Pediatr 1998;35:27-32.

28. Kapoor L, Randhawa VS, Deb M. Microbiological profile of neonatal septicemia in a pediatric care hospital in Delhi. J Commun Dis 2005;37:227-32.

29. Prashanth K, Badrinath S. Nosocomial infections due to Acinetobacter species: Clinical findings, risk and prognostic factors. Indian J Med Microbiol 2006;24:39-44.

30. Saleem AF, Ahmed I, Mir F, Ali SR, Zaidi AK. Pan-resistant Acinetobacter infection in neonates in Karachi, Pakistan. J Infect Dev Ctries 2009;4:30-7.

31. Shete VB, Ghadage DP, Muley VA, Bhore AV. Acinetobacter septicemia in neonates admitted to intensive care units. J Lab Physicians 2009;1:73-6.

32. De AS, Rathi MR, Mathur MM. Mortality audit of neonatal sepsis secondary to Acinetobacter. J Glob Infect Dis 2013;5:3-7.

33. Villegas MV, Hartstein AI. Acinetobacter outbreaks, 1977-2000. Infect Control Hosp Epidemiol 2003;24:284-95.

34. Signore C, Klebanoff M. Neonatal morbidity and mortality after elective cesarean delivery. Clin Perinatol 2008;35:361-71, vi.

35. Jawad A, Heritage J, Snelling AM, Gascoyne-Binzi DM, Hawkey PM. Influence of relative humidity and suspending menstrua on survival of Acinetobacter spp. On dry surfaces. J Clin Microbiol 1996;34:2881-7.

36. Sinha M, Srinivasa H, Macaden R. Antibiotic resistance profile and extended spectrum beta-lactamase (ESBL) production in Acinetobacter species. Indian J Med Res 2007;126:63-7.
37. Gladstone $\mathrm{P}$, Rajendran $\mathrm{P}, \mathrm{Brahmadathan} \mathrm{KN}$. Incidence of carbapenem resistant nonfermenting gram negative bacilli from patients with respiratory infections in the intensive care units. Indian J Med Microbiol 2005;23:189-91.

38. Hanberger H, Garcia-Rodriguez JA, Gobernado M, Goossens H, Nilsson LE, Struelens MJ, et al. Antibiotic susceptibility among aerobic gram-negative bacilli in intensive care units in 5 European countries. French and Portuguese ICU study groups. JAMA 1999;281:67-71.

39. Turner PJ, Greenhalgh JM; MYSTIC Study Group (Europe). The activity of meropenem and comparators against Acinetobacter strains isolated from European hospitals, 1997-2000. Clin Microbiol Infect 2003;9:563-7.

40. Turner PJ. Meropenem activity against European isolates: Report on the MYSTIC (Meropenem Yearly Susceptibility Test Information Collection) 2006 results. Diagn Microbiol Infect Dis 2008;60:185-92.

41. Perez F, Hujer AM, Hujer KM, Decker BK, Rather PN, Bonomo RA, et al. Global challenge of multidrug-resistant Acinetobacter baumannii. Antimicrob Agents Chemother 2007;51:3471-84.

42. Trottier V, Segura PG, Namias N, King D, Pizano LR, Schulman CI, et al. Outcomes of Acinetobacter baumannii infection in critically ill burned patients. J Burn Care Res 2007;28:248-54.

43. Peleg AY, Potoski BA, Rea R, Adams J, Sethi J, Capitano B, et al. Acinetobacter baumannii bloodstream infection while receiving tigecycline: A cautionary report. J Antimicrob Chemother 2007;59:128-31.

44. Chan JD, Graves JA, Dellit TH. Antimicrobial treatment and clinical outcomes of carbapenem-resistant Acinetobacter baumannii ventilator-associated pneumonia. J Intensive Care Med 2010;25:343-8.

45. Courvalin P. Antimicrobial drug resistance: "Prediction is very difficult, especially about the future". Emerg Infect Dis 2005;11:1503-6.

46. Pittet D. The Lowbury lecture: Behaviour in infection control. J Hosp Infect 2004;58:1-3. 